\title{
Phân biệt 3 mô hình đảm bảo chất lượng giáo dục đại học: Kiểm định chất lượng, đánh giá chất lượng và kiểm toán chất lượng
}

\author{
Nguyễn Hữu Cương* \\ Khoa Giáo dục, Truòng Đại hoc New South Wales, Australia, \\ High Street, Kensington, UNSW Sydney NSW 2052, Australia \\ Nhận ngày 26 tháng 5 năm 2016 \\ Chỉnh sửa ngày 08 tháng 9 năm 2016; Chấp nhận đăng ngày 15 tháng 03 năm 2017
}

\begin{abstract}
Tóm tắt: Bài viết này phân tích sự giống nhau và khác nhau giữa 3 mô hình đảm bảo chất lượng được sử dụng rộng rãi nhất trong các hệ thống giáo dục đại học trên thế giới: kiểm định chất lượng, đánh giá chất lượng và kiểm toán chất lượng. Trước hết, nghiên cứu trình bày những khái niệm liên quan đến đảm bảo chất lượng giáo dục đại học. Tiếp theo, nghiên cứu tập trung thảo luận chi tiết mỗi mô hình. Cuối cùng, bài viết đưa ra sự so sánh giữa 3 mô hình đảm bảo chất lượng này.
\end{abstract}

Tù khóa: Mô hình đảm bảo chất lượng, giáo dục đại học, kiểm định chất lượng, đánh giá chất lượng, kiểm toán chất lượng.

\section{1. Đặt vấn đề}

Đảm bảo chất lượng giáo dục đại học là một trong những vấn đề được các quốc gia, các tổ chức phi chính phủ, các tổ chức và mạng lưới khu vực và quốc tế quan tâm nhiều nhất trong những thập kỉ qua. Ở Việt Nam, vấn đề đảm bảo chất lượng giáo dục nói chung và đảm bảo chất lượng trong giáo dục đại học nói riêng đã được đề cập đển từ những năm chuyển giao giữa thế kỉ 20 và thế kỉ 21 , và được đặc biệt quan tâm trong vòng 10 năm vừa qua. Đã có nhiều mô hình đảm bảo chất lượng giáo dục đại học được triển khai ở nước ta, ví dụ như kiểm định chất lượng, đánh giá chất lượng, kiểm toán chất lượng, hoặc kiểm soát chất lượng. Bài viết này đi sâu vào nghiên cứu ba mô hình đảm bảo chất lượng được sử dụng rộng rãi nhất trên thế

ĐT.: +61405176886.

Email: cuongnh29@gmail.com giới hiện nay: kiểm định chất lượng, đánh giá chất lượng, và kiểm toán chất lượng.

Các thuật ngữ sử dụng trong bài viết này được dịch nguyên bản từ tiếng Anh. Các khái niệm cơ bản bao gồm: đảm bảo chất lượng (quality assurance), kiểm định chất lượng (accreditation), đánh giá chất lượng (assessment) và kiểm toán chất lượng (audit). Trước hết, bài viết trọng tâm vào tổng hợp các định nghĩa và đặc điểm chính của mỗi mô hình đảm bảo chất lượng. Sau đó, nghiên cứu so sánh những điểm giống nhau và khác nhau của ba mô hình này. Ngoài ra, ở mỗi mô hình đều có ví dụ về một số quốc gia trong khu vực và trên thế giới áp dụng mô hình đảm bảo chất lượng đó.

\section{2. Đảm bảo chất lượng giáo dục}

Để có thể bàn về đảm bảo chất lượng giáo dục thì trước hết phải hiểu chất lượng là gì. Tuy 
nhiên các nhà nghiên cứu cho rằng chất lượng là một khái niệm khó định nghĩa, khó xác định và khó đo lường. Harvey và Green (1993) đã đưa ra 5 quan niệm về chất lượng. Cụ thể là: (1) chất lượng là sự xuất sắc, (2) chất lượng là sự hoàn hảo, (3) chất lượng là sự phù hợp với mục tiêu, (4) chất lượng là sự đáng giá đồng tiền, và (5) chất lượng là giá trị chuyển đổi [1]. Trong số những quan nhiệm về chất lượng thì khái niệm chất lượng là sự phù hợp với mục tiêu được chấp nhận rộng rãi nhất [2].

Do có nhiều cách hiểu khác nhau về chất lượng, nên định nghĩa về đảm bảo chất lượng cũng rất đa dạng. Với quan niệm chất lượng là sự phù hợp với mục tiêu, Woodhouse cho rằng đảm bảo chất lượng là "các hệ thống, chính sách, thủ tục, quy trình, hành động và thái độ được cơ quan có thẩm quyền hoặc cơ sở giáo dục xác định, xây dựng và triển khai nhằm đạt được, duy trì, giám sát và củng cố chất lượng" [2].

Theo một tài liệu do UNESCO ấn hành thì đảm bảo chất lượng là một thuật ngữ rất rộng đề cập đến một quy trình đánh giá liên tục (bao gồm đánh giá, giám sát, đảm bảo, duy trì và nâng cao) chất lượng của một hệ thống giáo dục đại học, các cơ sở giáo dục và chương trình đào tạo [3].

Ngoài ra, theo Wilger (1997) thì đảm bảo chất lượng là một quá trình phức hợp mà qua đó trường đại học đảm bảo rằng chất lượng của các quy trình giáo dục được duy trì theo những tiêu chuẩn đã đề ra. Thông qua các hoạt động đảm bảo chất lượng, trường đại học có thể làm hài lòng chính nhà trường, sinh viên và những đối tượng khác ngoài nhà trường [4].

Qua ba định nghĩa trên, chúng ta có thể thấy những đặc điểm chính của đảm bảo chất lượng là: thư nhất, đảm bảo chất lượng tập trung vào quy trình, để từ đó khẳng định với cả những đối tượng bên trong và bên ngoài nhà trường rằng nhà trường có các quy trình để tạo ra sản phẩm đầu ra có chất lượng cao; thư hai, đảm bảo chất lượng tập trung vào chức năng giải trình và cải tiến chất lượng; thứ $b a$, đảm bảo chất lượng là một quá trình liên tục và thống nhất dựa trên các tiêu chí đánh giá và thông tin phản hồi.
Khi nói đến đảm bảo chất lượng, người ta thường nhắc đến 2 khái niệm đảm bảo chất lượng bên trong hay còn gọi là đảm bảo chất lượng nội bộ (internal quality assurance - IQA) và đảm bảo chất lượng bên ngoài (external quality assurance - EQA). Đảm bảo chất lượng bên trong liên quan đến các chính sách và cơ chế của mỗi cơ sở giáo dục hoặc chương trình đào tạo để đảm bảo rằng cơ sở giáo dục hoặc chương trình đào tạo đó thực hiện được các mục tiêu cũng như là các tiêu chuẩn áp dụng cho giáo dục đại học nói chung hoặc cho cho từng lĩnh vực nghề nghiệp nói riêng. Đảm bảo chất lượng bên ngoài liên quan đển các hoạt động của một đơn vị bên ngoài nhà trường, đó có thể là một tổ chức kiểm định chất lượng, đánh giá hoạt động của trường hoặc các chương trình đào tạo để quyết định liệu trường hoặc các chương trình đào tạo có đáp ứng các tiêu chuẩn đã thống nhất từ trước hay không. Các chuyên gia cũng cho rằng không nên xem đảm bảo chất lượng bên trong và đảm bảo chất lượng bên ngoài là đối lập hoặc mâu thuẫn với nhau. Ngược lại, chúng luôn tồn tại cùng với nhau. Đảm bảo chất lượng bên ngoài nên hỗ trợ và khuyến khích đảm bảo chất lượng bên trong [5].

Có nhiều mô hình hoặc cách tiếp cận đảm bảo chất lượng trong đại học. Trong đó, ba mô hình phổ biến nhất hiện nay là kiểm định chất lượng, đánh giá chất lượng và kiểm toán chất lượng.

\section{Kiểm định chất lượng giáo dục}

Kiểm định chất lượng là mô hình đảm bảo chất lượng được sử dụng rộng rãi nhất trong các hệ thống giáo dục đại học hiện nay. Kiểm định chất lượng bắt đầu được áp dụng ở Hoa Kỳ cách đây hơn 100 năm. Hội đồng kiểm định giáo dục đại học, Hoa Kỳ (CHEA) định nghĩa "kiểm định chất lượng là một quá trình xem xét chất lượng từ bên ngoài được giáo dục đại học tạo ra và sử dụng để đánh giá các trường cao đẳng, đại học và các chương trình đào tạo nhằm đảm bảo và cải tiến chất lượng”. Ở Hoa Kỳ thì kiểm định chất lượng là một quá trình dựa trên 
sự tin tưởng, tiêu chuẩn, bằng chứng, đánh giá và đồng cấp [6].

Ngoài ra, Vlăsceanu và các đồng nghiệp đã đưa ra một định nghĩa khá khát quát về kiểm định chất lượng (tài liệu do UNESCO ấn hành).

Kiểm định chất lượng là một quy trình mà một tổ chức công lập hoặc ngoài công lập hoặc tư nhân tiến hành đánh giá cơ sở giáo dục hoặc chương trình đào tạo để công nhận một cách chính thức cơ sở giáo dục hoặc chương trình đào tạo đạt được những tiêu chuẩn hoặc tiêu chí tối thiểu đã đề ra. Kết quả của kiểm định là quyết định công nhận đạt hoặc không đạt (có hoặc không) và cấp giấy chứng nhận quy định rõ thời gian có hiệu lực.

Quy trình kiểm định chất lượng thường bao gồm ba bước: (1) tự đánh giá của cơ sở giáo dục, (2) đánh giá ngoài của đoàn đánh giá ngoài do tổ chức kiểm định lựa chọn, và (3) thẩm định kết quả của hội đồng kiểm định [3].

Có hai loại hình kiểm định chất lượng là kiểm định cơ sở giáo dục (kiểm định trường) và kiểm định chương trình đào tạo. Kiểm định trường liên quan đến việc xem xét, đánh giá toàn bộ hoạt động của một cơ sở giáo dục đại học dựa trên một bộ tiêu chuẩn kiểm định trường. Kiểm định chương trình đào tạo (khóa đào tạo/ ngành đào tạo) là việc xem xét, đánh giá một phần của cơ sở giáo dục đại học liên quan trực tiếp đến một chương trình/ khóa đào tạo, và chú trọng vào các hoạt động chuyên môn. Kiểm định chương trình có thể được thực hiện với một bộ tiêu chuẩn kiểm định được dùng chung cho các chương trình đào tạo hoặc một bộ tiêu chuẩn được xây dựng cho một chương trình đào tạo cụ thể.

Trong các quốc gia áp dụng kiểm định chất lượng thì Hoa Kỳ được biết đến nhiều nhất với kiểm định trường và kiểm định chương trình. Ở Châu Ầu khoảng một nửa số tổ chức đảm bảo chất lượng bên ngoài thực hiện kiểm định chương trình, trong khi đó chỉ có $22 \%$ thực hiện kiểm định trường. Ví dụ một số quốc gia ở
Châu Âu áp dụng mô hình kiểm định như Đức, Pháp, Hà Lan, và Áo [7].

Trong khu vực Đông Nam Á, những quốc gia áp dụng kiểm định chất lượng bao gồm Brunei, Campuchia, Indonesia, Lào, Malaysia, Đông Timo và Việt Nam [8].

\section{4. Đánh giá chất lượng giáo dục}

Woodhouse định nghĩa đánh giá chất lượng là sự đánh giá đưa đến kết quả điểm số, có thể là con số (ví dụ 1 đến 4 ), tỉ lệ phần trăm, chữ số (ví dụ $\mathrm{A}$ đến $\mathrm{F}$ ) hoặc miêu tả (ví dụ xuất sắc, tốt, thỏa mãn, không thỏa mãn). Đánh giá có thể đưa ra giới hạn đỗ/trượt theo một phổ điểm (hoặc chỉ đơn giản là thang điểm 2 số). Đánh giá chất lượng đưa ra câu hỏi "kết quả của bạn tốt thế nào?" [2].

Theo SEAMEO RIHED (2012) thì đánh giá chất lượng phân tích kết quả đầu ra. Kết quả của đánh giá chất lượng dựa vào điểm (có thể dưới dạng con số, chữ cái hoặc mô tả). Đánh giá chất lượng thường xem xét các dữ liệu chỉ số thực hiện thể hiện dưới hình thức định lượng. Kết quả của một đợt đánh giá chất lượng là giấy chứng nhận đạt mức đánh giá hoặc báo cáo đánh giá ngoài [8].

Cũng giống như kiểm định chất lượng, có đánh giá chất lượng cơ sở giáo dục và đánh giá chất lượng chương trình đào tạo. Đánh giá chất lượng cũng được sử dụng khá phổ biến ở Châu Âu, trong đó đánh giá chất lượng chương trình đào tạo được sử dụng phổ biến hơn với khoảng $53 \%$ các tổ chức đảm bảo chất lượng của Châu Âu. Trong khi đó đánh giá chất lượng cơ sở giáo dục chỉ chiếm $22 \%$. Các nước sử dụng cách tiếp cận đánh giá như Đan Mạch, Phần Lan và Vương quốc Anh [7].

Những quốc gia trong khu vực Đông Nam Á áp dụng mô hình đánh giá chất lượng như Indonesia (cũng áp dụng kiểm định), Singapore và Thái Lan. Mô hình đảm bảo chất lượng mà AUN-QA đang sử dụng cũng là đánh giá chất lượng [8]. 


\section{Kiểm toán chất lượng giáo dục}

Kiểm toán chất lượng là một cách tiếp cận đảm bảo chất lượng khá đặc biệt. Không giống như kiểm định hoặc đánh giá tập trung vào xem xét chất lượng hoặc chỉ số thực hiện, kiểm toán kiểm tra chất lượng của các cơ chế đảm bảo chất lượng.

Cụ thể, kiểm toán chất lượng xem xét quy trình mà cơ sở giáo dục hoặc chương trình đào tạo thực hiện để đảm bảo chất lượng và nâng cao chất lượng. Kiểm toán đánh giá xem quy trình đảm bảo chất lượng có hợp lý không và có thực sự đang được triển khai không. Kiểm toán chất lượng nhìn vào toàn hệ thống hoặc toàn bộ các quy trình để đạt được chất lượng chứ không phải tập chung vào chất lượng. Một đợt kiểm toán có thể được thực hiện bởi những người (gọi là kiểm toán viên) mà họ không có chuyên môn liên quan trực tiếp đến lĩnh vực được kiểm toán.

Kiểm toán chất lượng tập trung vào trả lời các câu hỏi "làm thế nào" hoặc "quy trình đảm bảo chất lượng có hiệu quả không?" Kết quả của một đợt kiểm toán là báo cáo kiểm toán [3, $5,8]$.

Theo Woodhouse (1999) thì kiểm toán chất lượng xác minh 3 vấn đề sau:

- Sự phù hợp của các quy trình đảm bảo chất lượng với những mục tiêu đề ra;

- Sự tuân thủ các quy trình đảm bảo chất lượng đã được lập kế hoạch với những mục tiêu đề ra; và

- Sự hiệu quả của các hoạt động để đạt được những mục tiêu đề ra [2].

Kiểm toán chất lượng không được nhiều hệ thống giáo dục đại học trên thế giới sử dụng. Ở Châu Âu chỉ có $28 \%$ các tổ chức đảm bảo chất lượng thực hiện kiểm toán chất lượng cở sở đào tạo. Còn kiểm toán chất lượng chương trình đào tạo thì không phổ biến. Các nước áp dụng mô hình kiểm toán như Iceland, Ireland và Australia (trước năm 2012). Một số quốc gia trong khu vực Đông Nam Á cũng áp dụng mô hình kiểm toán chất lượng như Philippines (áp dụng cả kiểm định và đánh giá), Singapore (áp dụng cả đánh giá) và Thái Lan (áp dụng đánh giá là chủ yếu) [7].

\section{So sánh 3 mô hình đảm bảo chất lượng}

Qua những phần trình bày ở trên chúng ta có thể thấy rằng cả ba cách tiếp cận đảm bảo chất lượng: kiểm định, đánh giá và kiểm toán đều có chung một mục đích là để đảm bảo và nâng cao chất lượng của một cơ sở giáo dục hoặc một chương trình đào tạo. Tuy nhiên, chúng có một số điểm khác biệt ở quy trình hoặc sản phẩm đầu ra.

Với kiểm định chất lượng và đánh giá chất lượng thì kết quả đầu ra có thể giống nhau (đỗ/trượt và giấy chứng nhận). Còn sự khác biệt lớn nhất giữa hai cách tiếp cận chất lượng này là kiểm định trọng tâm vào xem xét về việc đạt chuẩn tối thiểu, còn đánh giá tập trung vào phân tích kết quả đầu ra.

Kiểm toán chất lượng khác với kiểm định chất lượng và đánh giá chất lượng ở đối tượng đánh giá. Đối tượng quan tâm của kiểm định và đánh giá là chất lượng, còn đối tượng quan tâm của kiểm toán là quy trình tạo nên chất lượng. Ngoài ra, kết quả đầu ra của kiểm toán cũng khác so với kết quả đầu ra của kiểm định hoặc đánh giá. Kết quả của một đợt kiểm toán chất lượng là báo cáo kiểm toán tập trung vào mô tả và khuyến nghị, còn kết quả của một đợt kiểm định hoặc đánh giá là sự công nhận (đạt/không đạt) hoặc điểm số và giấy chứng nhận. Những đặc điểm chính của kiểm định chất lượng, đánh giá chất lượng và kiểm toán chất lượng được tóm tắt trong Bảng 1.

Ngoài ra, Woodhouse (1999) cho rằng trong 5 bước của đảm bảo chất lượng, bao gồm: 1 Mục tiêu thích hợp, 2-Kế hoạch hợp lí, 3-Hành động phù hợp, 4-Hành động hiệu quả, 5-Kết quả đo lường được, thì không một mô hình đảm bảo chất lượng nào như trình bày ở trên bao quát được tất cả các bước. Khi áp dụng vào thực tế thì kiểm định bao quát được từ bước 1 đến bước 4 , kiểm toán từ bước 2 đến bước 4 , đánh giá trọng tâm vào bước 5 và có thể bao quát từ bước 2 đến bước 4 . Tóm tắt nghiên cứu này của Woodhouse được trình bày ở Hình 1 . 
Bảng 1. So sánh kiểm định, đánh giá và kiểm toán

\begin{tabular}{|c|c|c|c|}
\hline & Kiểm định & Đánh giá & Kiểm toán \\
\hline $\begin{array}{l}\text { Những đặc điểm } \\
\text { chính }\end{array}$ & $\begin{array}{l}\text { Tập trung đánh giá để công nhận đạt } \\
\text { hoặc không đạt tiêu chuẩn tối hiểu }\end{array}$ & $\begin{array}{l}\text { Tập trung xem xét } \\
\text { kết quả đầu ra }\end{array}$ & $\begin{array}{l}\text { Tập trung xem xét quy } \\
\text { trình }\end{array}$ \\
\hline Kết quả & $\begin{array}{l}\text { - Công nhận đạt hoặc không đạt } \\
\text { - Giấy chứng nhận }\end{array}$ & $\begin{array}{l}\text { - Báo cáo đánh } \\
\text { giá với mức đánh } \\
\text { giá (ví dụ điểm } \\
\text { số) } \\
\text { - Có thể có giấy } \\
\text { chứng nhận }\end{array}$ & $\begin{array}{l}\text { Báo cáo kiểm toán (tập } \\
\text { trung vào mô tả và } \\
\text { khuyến nghị) }\end{array}$ \\
\hline $\begin{array}{l}\text { Câu hỏi liên } \\
\text { quan }\end{array}$ & Chất lượng có thực sự tốt không? & $\begin{array}{l}\text { Kết quả đầu ra tốt } \\
\text { như thế nào? }\end{array}$ & $\begin{array}{l}\text { Quy trình đảm bảo chất } \\
\text { lượng có hiệu quả không? }\end{array}$ \\
\hline
\end{tabular}

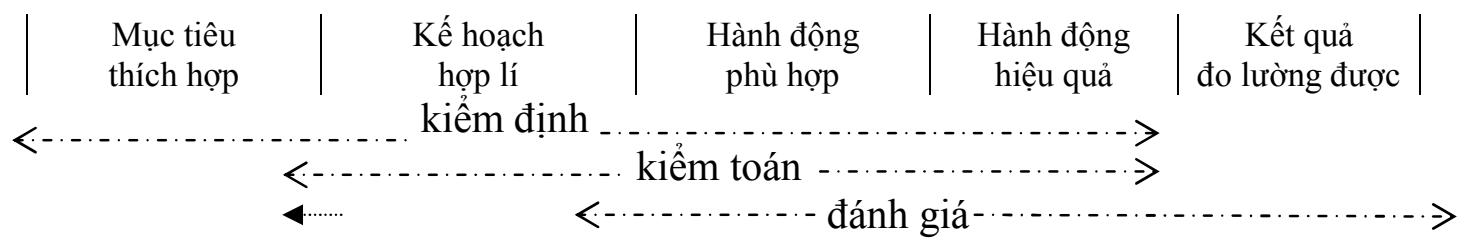

Hình 1. Kiểm định, kiểm toán, đánh giá trong quy trình 5 bước của đảm bảo chất lượng (nguồn: Woodhouse, 1999) [2].

Tuy nhiên, Woodhouse cũng cho rằng mặc dù kiểm định, đánh giá và kiểm toán có những điểm đặc trưng khác nhau, ba mô hình đảm bảo chất lượng này có thể trùng lặp hoặc hòa nhập với nhau. Bất kì một nỗ lực nào để đưa ra một định nghĩa chính xác hoặc phân biệt tuyệt đối giữa ba mô hình này có thể càng gia tăng sự rắc rối, khó hiểu bởi vì hầu hết các thuật ngữ ở đây đều liên quan đến quy trình xem xét hoặc đánh giá [2].
Ngoài ra, các nghiên cứu cũng chỉ ra rằng hầu hết các quốc gia đều sử dụng nhiều hơn một cách tiếp cận về đảm bảo chất lượng. Ví dụ có một số nước sử dụng đánh giá và kiểm định, một số nước áp dụng kiểm toán và đánh giá hoặc sử dụng cả ba mô hình đảm bảo chất lượng [8]. Bảng 2 tóm tắt các mô hình đảm bảo chất lượng được sử dụng ở các nước ASEAN.

Bảng 2. Các mô hình đảm bảo chất lượng ở một số nước ASEAN (nguồn: SEAMEO RIHED) [8]

\begin{tabular}{ll}
\hline Quốc gia & Mô hình $\mathbf{~} \mathbf{B C L}$ \\
\hline Brunei & Kiểm định trường và chương trình \\
Campuchia & Kiểm định trường và chương trình \\
Indonesia & Kiểm định trường và chương trình + đánh giá trường và chương trình \\
Malaysia & Kiểm định trường và chương trình \\
Phillipines & Kiểm định trường và chương trình + đánh giá chương trình + kiểm toán \\
Singapore & Kiểm toán các trường công lập + đánh giá các trường tư thục \\
Thái Lan & Đánh giá trường và chương trình + kiểm toán trường và chương trình \\
Việt Nam & Kiểm định trường và chương trình \\
\hline
\end{tabular}

\section{Kết luận}

Kiểm định, đánh giá và kiểm toán là 3 cách tiếp cận về đảm bảo chất lượng được sử dụng phổ biến nhất trong các hệ thống giáo dục đại họ̣c trên thế giới hiện nay. Cả ba mô hình này đều trọng tâm vào củng cố và cải tiến chất lượng. Tuy nhiên, mỗi cách tiếp cận có những đặc điểm, quy trình và kết quả đầu ra khác nhau. Do đó, mỗi mô hình cũng có những thế 
mạnh và ưu điểm riêng, cũng như những hạn chế nhất định.

Việc triển khai thành công mỗi cách tiếp cận về đảm bảo chất lượng phụ thuộc vào nhiều yếu tố như bối cảnh quốc gia, văn hóa, hoặc sự phát triển của hệ thống giáo dục đại học. Để áp dụng các mô hình đảm bảo chất lượng này một cách hiệu quả, các nước cần nghiên cứu kĩ mỗi mô hình. Một mô hình có thể vận hành tốt ở quốc gia này, nhưng có thể sẽ không hiệu quả khi được triển khai ở quốc gia khác.

\section{Tài liệu tham khảo}

[1] Harvey, L., \& Green, D., Defining quality, Assessment and Evaluation in Higher Education, Vol.18, No.1 (1993) 9.

[2] Woodhouse, D., Quality and quality assurance, Quality and Internationalisation in Higher Education, OECD-IMHE, Paris, (1999) 29.
[3] Vlăsceanu, L., Grünberg, L., \& Pârlea, D., Quality assurance and accreditation: a glossary of basic terms and definitions, UNESCOCEPES, Bucharest, 2007.

[4] Wilger, A., Quality assurance in higher education: a literature review, Stanford University, Stanford, CA, 1997.

[5] UNESCO-IIEP, External quality assurance: options for higher education managers, UNESCO-IIEP, Paris, 2006.

[6] Eaton, J., An overview of U.S. accreditation, Council for Higher Education Accreditation, Washington DC, 2015.

[7] Kis, V., Quality assurance in tertiary education: current practices in OECD countries and a literature review on potential effects, OECD, Paris, 2005.

[8] SEAMEO RIHED, A study on quality assurance models in Southeast Asian countries: towards a southeast Asian quality assurance framework, SEAMEO RIHED, Bangkok, 2012.

\title{
Distinguishing Three Quality Assurance Models in Higher Education: Accreditation, Assessment and Audit
}

\author{
Nguyen Huu Cuong \\ School of Education, the University of New South Wales, Australia, \\ High Street, Kensington, UNSW Sydney NSW 2052, Australia
}

\begin{abstract}
This paper analyses the similarities and differences between the world's three most widely used higher education quality assurance models: accreditation, assessment and audit. Firstly, the paper presents an overview of the concepts related to higher education quality assurance. Next, each of the three models is discussed in detail. Finally, the paper compares the three quality assurance models.
\end{abstract}

Keywords: Quality assurance model, higher education, accreditation, assessment, audit. 